\title{
Blood pressure management in stroke
}

Five new things

\author{
Ritvij Bowry, MD \\ Digvijaya D. Navalkele, MD, MPH \\ Nicole R. Gonzales, MD
}

\begin{abstract}
Summary
Hypertension is a major modifiable risk factor for stroke, with an estimated $51 \%$ of stroke deaths being attributable to high systolic blood pressure globally. ${ }^{1,2}$ The management of hypertension in stroke is determined by timing, the type of stroke, use of thrombolysis, concurrent medical conditions, and pharmacologic variables. We highlight the details of elevated blood pressure management in the hyperacute/acute, subacute, and chronic stages of ischemic stroke and intracerebral hemorrhage.
\end{abstract}

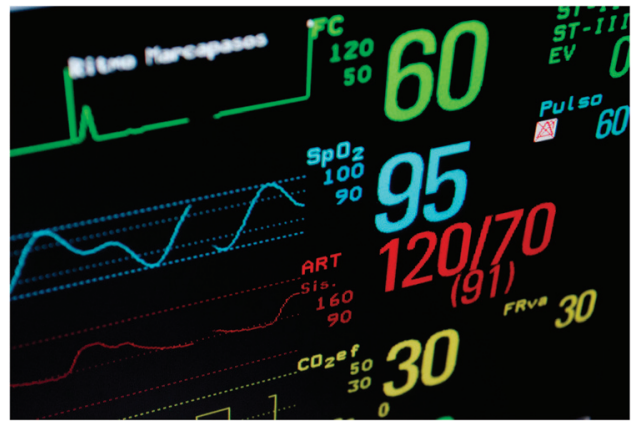
he relationship between hypertension and stroke is dynamic and multifaceted. Be it in the context of managing ischemic or hemorrhagic stroke, selecting an appropri- ate blood pressure (BP) agent involves integration of several issues that must be rec- ognized in order to formulate an effective strategy for BP control.

\section{Blood pressure, ischemic stroke, and thrombolysis}

More than $60 \%$ of patients with acute ischemic stroke (AIS) present with elevated BP within 1 hour of symptom onset. ${ }^{3}$ Elevated BP can affect thrombolytic eligibility and has been associated with delay in administration of IV tissue plasminogen activator (IV tPA). ${ }^{4}$ Since $<30 \%$ of patients have door-to-treatment times less than the recommended 60 minutes, timely management of elevated BP is crucial when patients are otherwise eligible for IV tPA. ${ }^{5}$ Earlier thrombolytic treatment of patients with AIS is not only associated with more frequent independent ambulation at discharge and discharge to home, but is also associated with reduced mortality and symptomatic intracerebral hemorrhage $(\mathrm{sICH}){ }^{6}$

One factor that has been associated with delays in treatment times is the need for prethrombolytic BP goal of $<185 / 110 \mathrm{~mm} \mathrm{Hg},{ }^{7,8}$ a target extrapolated from prior studies of thrombolysis in acute myocardial infarction. ${ }^{9} 10$ In cases where such a target is not achieved, tPA may even be withheld, given the association of elevated BP and risk of sICH leading to poor clinical outcomes. ${ }^{11}$ Withholding tPA based solely on persistently uncontrolled BP, however, can lead to as many as $10 \%$ of otherwise eligible patients not receiving tPA. This is a significant

University of Texas Health Sciences Center at Houston.

Funding information and disclosures are provided at the end of the article. Full disclosure form information provided by the authors is available with the full text of this article at Neurology.org/cp.

Correspondence to: Ritvij.Bowry@uth.tmc.edu 


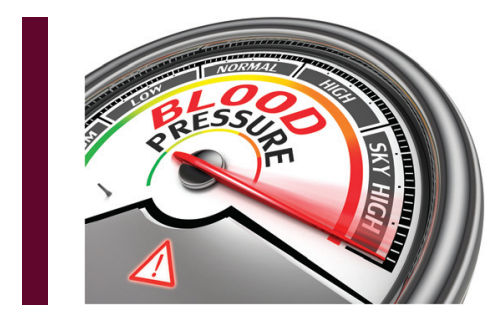

\section{Since $<30 \%$ of patients have door-to-treatment times less than the recommended 60 minutes, timely management of elevated BP is crucial when patients are otherwise eligible for IV tPA.}

number considering that $<10 \%$ of patients meet the current eligibility criteria for the use of IV tPA within 4.5 hours. ${ }^{12}$ Thus, a proficient attempt must be made to reduce BP to the thrombolytic range, even if it involves using multiple BP agents or continuous infusions. Keeping in mind the importance of not making the patient relatively hypotensive, ${ }^{13}$ the safety of aggressively lowering BP to $<185 / 110 \mathrm{~mm} \mathrm{Hg}$ using continuous IV BP agents has been demonstrated to be safe in patients eligible for IV tPA presenting within 3 hours of symptom onset without adverse effect on clinical outcomes. ${ }^{14} \mathrm{With}$ a synchronized effort, we propose that most thrombolytic eligible patients can have their BP brought down to the treatment range quickly enough so that thrombolysis remains an option for this debilitating disease.

The addition of intra-arterial (IA) therapy to the AIS treatment regimen requires special attention to BP with multiple variables involved. While uncertainty remains around optimal BP parameters, pending more definitive data, there are some concepts that can be considered in this scenario.

Prior to the procedure, $\mathrm{BP}<180 / 100 \mathrm{~mm} \mathrm{Hg}$ is often designated, especially if IA lysis with tPA is planned..$^{15}$ During the procedure, target BP within $10 \%-20 \%$ of the admission BP is a reasonable goal if IA recanalization is used as monotherapy, ${ }^{15}$ or $<180 / 105 \mathrm{~mm} \mathrm{Hg}$ if used adjunctively with IV tPA. Extreme caution should be taken to avoid relative hypotension during the procedure, especially when general anesthesia is used. ${ }^{16}$ In addition, systolic BP (SBP) $>140 \mathrm{~mm} \mathrm{Hg}$ is generally targeted during the procedure, as BP below this threshold has been shown to be independently predictive of poor neurologic outcomes after endovascular treatment. ${ }^{17}$ Postprocedure, $\mathrm{BP}$ can be titrated according to the degree of arterial recanalization and the patient's neurologic examination. If complete recanalization is achieved, then goal BP may be lowered to a SBP of $120-140 \mathrm{~mm} \mathrm{Hg}$ to lower the risk of reperfusion hemorrhage. In cases of partial recanalization, it is reasonable to maintain SBP up to $185 \mathrm{~mm} \mathrm{Hg}$ for $24-48$ hours in order to augment collateral blood flow and clear emboli from distal vasculature, ${ }^{15,18}$ unless the patient has received IV tPA. BP must ultimately be optimized to minimize the rate of sICH and reperfusion injury and to promote adequate cerebral perfusion. ${ }^{16}$

\section{BP and ischemic stroke when thrombolysis is not an option}

In cases of AIS where thrombolysis is not a consideration, there is uncertainty surrounding the optimal management of BP in the acute setting. ${ }^{8}$ The decision to reduce BP in the first 24 hours of a stroke must take into consideration the potential of compromising collateral blood flow and hastening the interval to infarction (range 6-18 hours after large vessel ischemic stroke), ${ }^{19}$ vs the potential for adverse systemic effects as a result of persistently elevated BP. As a result, it is best to observe current guidelines, which recommend a $15 \%$ reduction within the first 24 hours of ischemic stroke only in cases where BP exceeds 220/120 mm Hg. ${ }^{8}$

Given the association between clinical outcomes in AIS and the direction and magnitude of BP changes over the first $24-48$ hours, ${ }^{20}$ antihypertensive therapy is routinely initiated 24 hours after AIS. Although it is intuitive that BP control at all timepoints after AIS would be beneficial, recent evidence has suggested that $\mathrm{BP}$ control beyond 15 hours from onset of an ischemic stroke (even up to 2 weeks) may have little effect on clinical outcome. ${ }^{21,22}$ Given the limitations in these studies $^{23}$ and until more definitive evidence is available, our practice is to gently normalize BP during hospitalization. Regardless of when BP medication is resumed, a management strategy must ensure a patient's neurologic stability prior to $\mathrm{BP}$ control and minimize $\mathrm{BP}$ variability, ${ }^{24}$ given the association of wide BP fluctuations and poor outcomes at 1 and 3 months. ${ }^{25}$ 


\section{Whereas the timing of BP control is}

complicated in itself, other factors such as stroke subtype and cerebrovascular anatomy must also be taken into account.

\section{Specific goals for specific conditions}

Whereas the timing of BP control is complicated in itself, other factors such as stroke subtype and cerebrovascular anatomy must also be taken into account.

Small-vessel disease The Secondary Prevention of Small Subcortical Strokes trial demonstrated that lowering SBP to a goal $<130 \mathrm{~mm} \mathrm{Hg}$ vs a range of $130-149 \mathrm{~mm} \mathrm{Hg}$ at least 2 weeks after MRI-confirmed lacunar strokes was not only safe and well-tolerated, but also reduced the rate of all stroke (hazard ratio [HR] 0.81, 95\% confidence interval [CI] 0.64$1.03, p=0.08$ ), disabling or fatal strokes (HR 0.81, 0.53-1.23, $p=0.32$ ), other major vascular events (HR 0.84, 0.68-1.04, $p=0.32$ ), and intracerebral hemorrhage (ICH) (HR $0.37,0.15-0.95, p=0.03$ ) over a period of $3-4$ years. ${ }^{26}$ This study excluded patients with alternate stroke etiologies, such as ipsilateral carotid stenosis, high-risk cardioembolic strokes, and disabling strokes, where other clinical variables may also play in role in devising a BP management plan.

Intracranial atherosclerosis Regarding recurrent strokes related to severe atherosclerotic disease of large intracranial vessels, prior evidence has indicated an increased stroke risk with elevated diastolic BP in patients with $\geq 70 \%$ stenosis of a major intracranial artery. 27,28 Recent evidence, however, has demonstrated the importance of SBP control as well in preventing strokes in a similar cohort of patients. In this study, patients who had nondisabling strokes or TIAs attributable to $70 \%-99 \%$ stenosis of a major intracranial artery (carotid artery, middle cerebral artery, vertebral artery, or basilar artery), and in whom an aggressive medical management strategy was employed (goal SBP $<140[<130 \mathrm{~mm} \mathrm{Hg}$ in diabetes], low-density lipoprotein [LDL] $<70 \mathrm{mg} / \mathrm{dL}$, dual antiplatelet therapy with aspirin and clopidogrel for 90 days, and lifestyle modifications), had fewer cerebrovascular events in the subsequent 30 days compared with those who had a similar medical management strategy plus intracranial angioplasty and stenting. This beneficial effect was sustained in the long term over a median duration of 32.4 months (absolute risk reduction from medical treatment was $8.9 \%$ at 30 days and $9.0 \%$ at 3 years). While the findings from this study are partly attributable to the nature of the management strategy of using dual antiplatelet agents and targeting a lower LDL goal, it is notable that $70 \%$ of patients at 1 year continued to meet the target SBP goal of $<140 \mathrm{~mm} \mathrm{Hg}$ (compared to $34 \%$ at enrollment). ${ }^{29}$ The fact that such BP goals were attainable within a framework of other key prevention strategies overseen primarily by neurologists and study coordinators is a testament to the important role that all providers play in taking care of stroke patients, including both general neurologists and stroke specialists.

\section{Acute reduction of $\mathrm{BP}$ in $\mathrm{ICH}$ is safe}

One aspect of the controversy regarding the safety of rapidly lowering BP in acute hemorrhagic stroke has been a concern for acute ischemia resulting from decreased cerebral blood flow (CBF) as a result of rapid BP reduction. Neuroimaging evidence has suggested otherwise, with PET studies showing neither a reduction in global and perihematomal CBF after acute reductions in mean arterial pressures nor a significant compromise in cerebral blood volume as a result of lowering SBP to a target of $<150 \mathrm{~mm} \mathrm{Hg} \cdot{ }^{30, \mathrm{e}}$ Similar markers of ischemia, such as diffusion restriction on MRI in the perihematomal region, have been attributed to nonischemic pathologies, such as vasogenic edema, inflammation, or mechanical cellular injury. ${ }^{\mathrm{e} 2, e 3}$

Supplemental Data

Neurology.org/cp 


\title{
Table Blood pressure management and stroke
}

\author{
Ischemic stroke and TIA 8,26,28,29,e18
}

Acute setting

Patients eligible for acute reperfusion

For BP >185/110 mm Hg: administer labetalol 10-20 mg over 1-2 minutes, may repeat 1 time; or start nicardipine $5 \mathrm{mg} / \mathrm{h} \mathrm{IV}$, titrate up by $2.5 \mathrm{mg} / \mathrm{h}$ every 5-15 minutes for maximum $15 \mathrm{mg} / \mathrm{h}$; or add other agents (hydralazine, enalaprilat)

During and after reperfusion therapy BP goal $\leq 180 / 105 \mathrm{~mm} \mathrm{Hg}$

Patients not eligible for acute reperfusion therapy

For SBP $>220 \mathrm{~mm} \mathrm{Hg}$ or DBP 121-140 mm Hg, administer labetalol IV or nicardipine as IV infusion, aiming for a 10\%-15\% reduction of BP

If DBP $>140 \mathrm{~mm} \mathrm{Hg}$, give sodium nitroprusside as IV infusion, titrating the dose for a $10 \%-15 \%$ reduction of $\mathrm{BP}$

\section{Subacute setting}

Previously untreated patients with SBP Initiate BP therapy (Class I; Level of evidence B)

$\geq 140 \mathrm{~mm} \mathrm{Hg}$ or DBP $\geq 90 \mathrm{~mm} \mathrm{Hg}$

Patients with SBP $<140 \mathrm{~mm} \mathrm{Hg}$ and DBP Initiation of BP therapy is of uncertain benefit (Class Ilb; Level of evidence C) $<90 \mathrm{~mm} \mathrm{Hg}$

Previously treated patients with known Resume BP therapy (Class I; Level of evidence A)

hypertension

Reasonable to achieve $\mathrm{BP}<140 / 90 \mathrm{~mm} \mathrm{Hg}$ as a target if patients do not have specific indications as below (Class Ila; Level of evidence B)

\section{Specific indications}

\section{Recent lacunar stroke}

Intracranial atherosclerosis $\mathbf{5 0 \% - 9 9 \%}$ stenosis of a major intracranial artery)
SBP $<130 \mathrm{~mm} \mathrm{Hg}$ (Class Ilb; Level of evidence B)

Target SBP $<140$ mm Hg (Class I; Level of evidence B)

\section{Intracerebral hemorrhage $\mathrm{e}^{\mathrm{e}, \mathrm{e}, \mathrm{e}, \mathrm{e} 11}$}

When SBP is $150-220 \mathrm{~mm} \mathrm{Hg}$, acute

lowering to $140 \mathrm{~mm} \mathrm{Hg}$ is reasonable

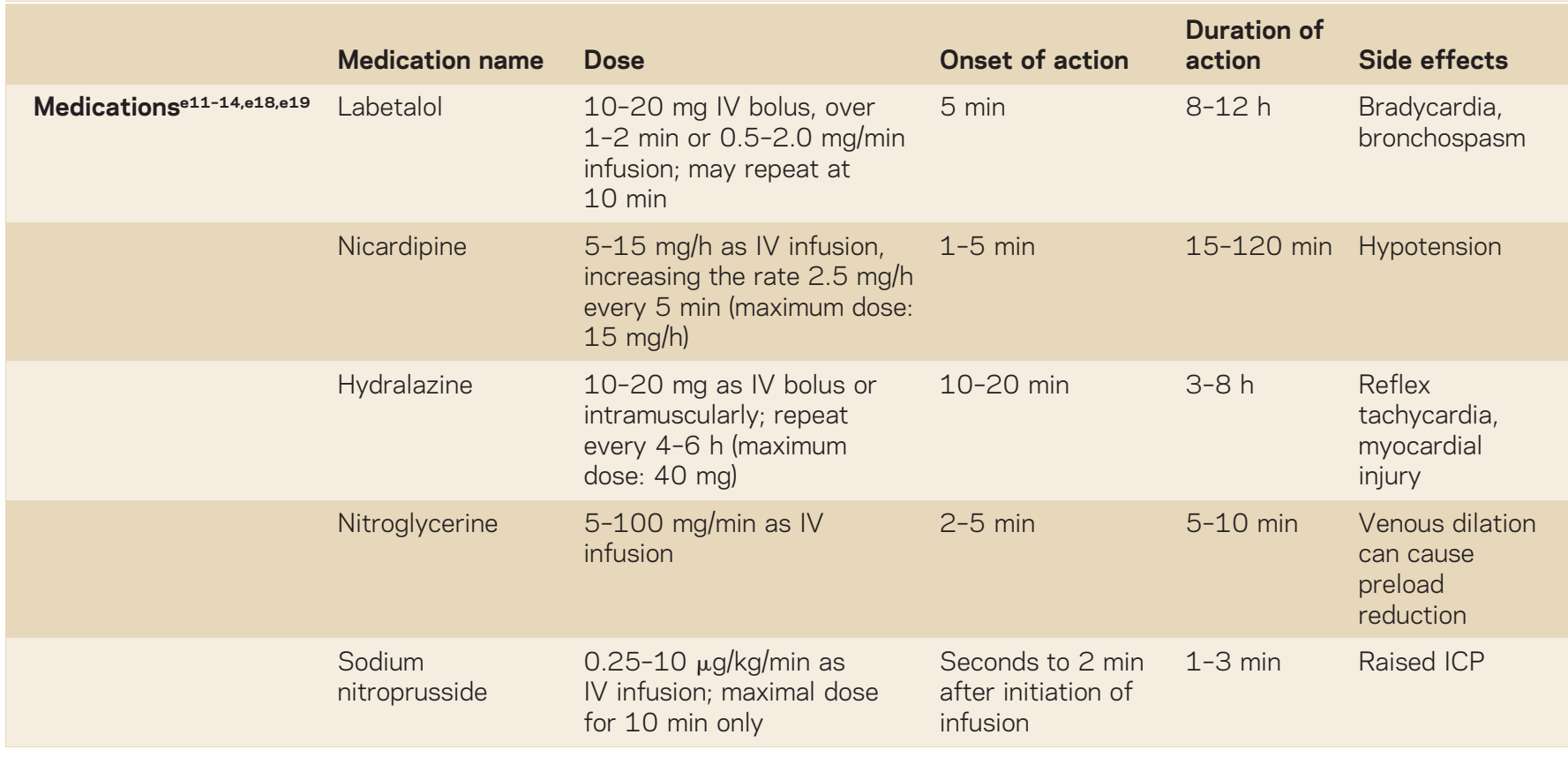




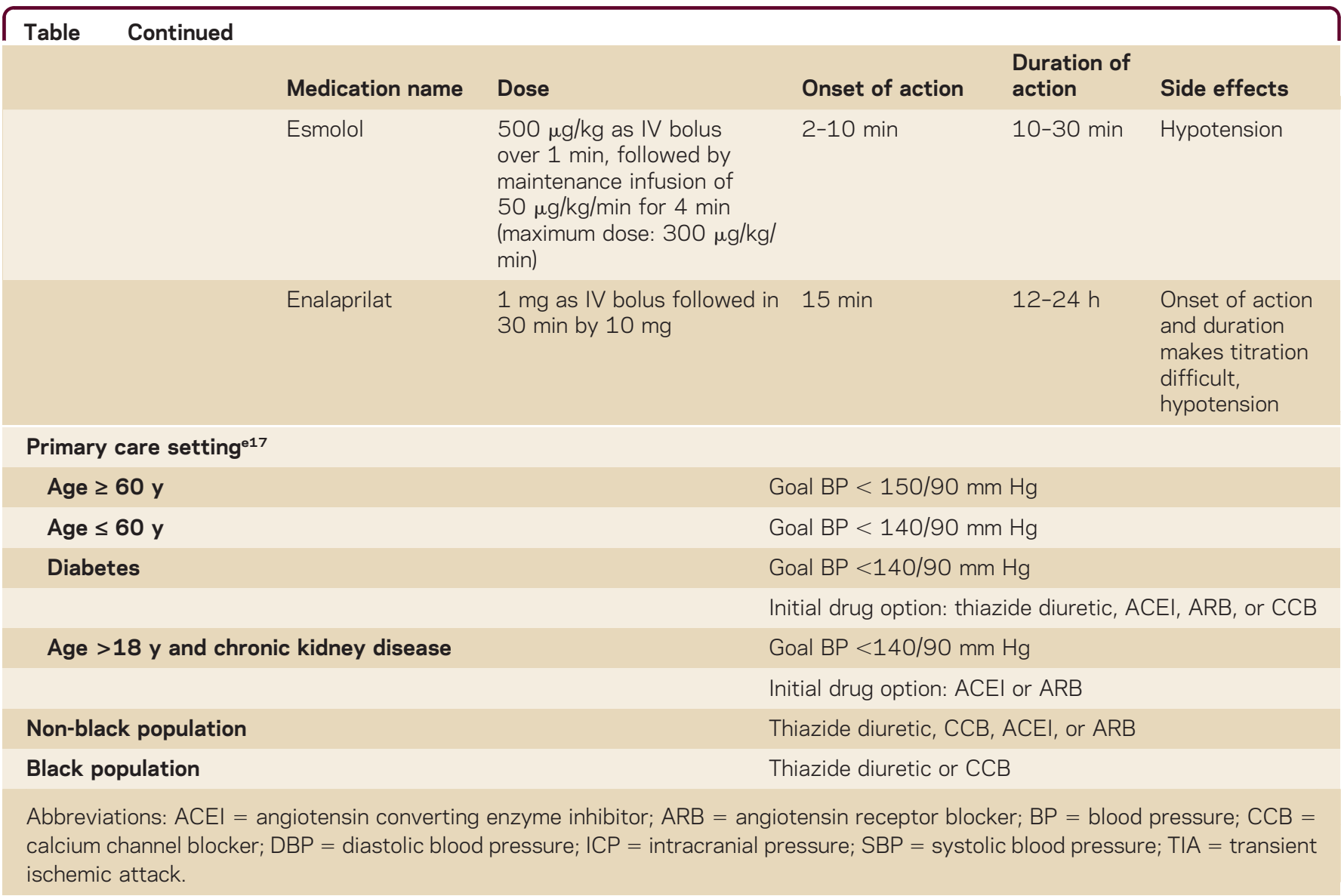

The second component of this debate has focused on the clinical safety of acutely lowering $\mathrm{BP}$, since elevated $\mathrm{BP}$ after $\mathrm{ICH}$ is not only predictive of outcome but is associated with recurrent hemorrhage. ${ }^{\mathrm{e}, \mathrm{e} 5}$ The safety and effect on hematoma growth of lowering BP was demonstrated in the Intensive Blood Pressure Reduction in Acute Cerebral Hemorrhage (INTERACT) trials, which not only supported the safety of an intensive BP-lowering strategy (SBP range of $130-140 \mathrm{~mm} \mathrm{Hg})^{\mathrm{e} 6}$ but also showed a reduction of hematoma enlargement (a factor associated with poor outcome) at 24 and 72 hours in patients who had intensive BP lowering vs a guideline-based management (SBP $<180 \mathrm{~mm} \mathrm{Hg}$ ) when treatment was initiated within 6 hours of symptom onset. ${ }^{\mathrm{e} 7} \mathrm{~A}$ similar safety trend was also demonstrated with the acute reduction of SBP to a goal $110-140 \mathrm{~mm} \mathrm{Hg}$ within 6 hours of symptom onset of an acute $\mathrm{ICH}$ in the Antihypertensive Treatment of Acute Cerebral Hemorrhage (ATACH) trial. $^{\text {e8 }}$

With regards to clinical efficacy, no significant reduction was seen in the primary outcomes of the rate of death or major disability as a result of early intensive BP reduction in the INTERACT-2 trial (odds ratio [OR] with intensive treatment $0.87 ; 95 \%$ CI $0.75-1.01 ; p=0.06$ ). An ordinal analysis of the modified Rankin score, however, did suggest that intensive BP treatment improved functional outcomes (OR for greater disability $0.87 ; 95 \%$ CI $0.77-1.00 ; p=0.04$ ). There was also meaningful evidence for better physical and psychological well-being in patients in whom the $\mathrm{BP}$ was intensively lowered, without a concomitant increase in the rate of death or serious adverse events. ${ }^{\mathrm{e}, \mathrm{e} e}$ The ATACH II trial that is currently investigating the effectiveness and safety of lowering SBP to a goal $<140 \mathrm{~mm} \mathrm{Hg}$ within 4.5 hours will provide additional information on whether an antihypertensive treatment strategy targeting a more stringent time metric is safe and can improve clinical outcomes. ${ }^{10}$ Based on the INTERACT and ATACH trials, the current American Heart Association recommendations endorse the safety of acutely 


\section{Blood Pressure Management In Stroke: Five new things}

- Patients who present with acute ischemic stroke and are otherwise eligible for IV tPA (except for severely elevated BP) can become thrombolytic candidates with rapid and efficient BP treatment.

- When thrombolysis is not an option, acute management of $\mathrm{BP}$ is a balancing act between maintaining cerebral perfusion and avoiding systemic adverse events due to persistently elevated BP.

- In the acute setting of $\mathrm{ICH}$, rapidly lowering $\mathrm{BP}$ to $<140 / 90 \mathrm{~mm} \mathrm{Hg}$ is safe and may be associated with improved radiographic and clinical outcomes.

- In the hyperacute setting of both ischemic and hemorrhagic stroke, initiation of continuous IV administration of newer agents may achieve treatment goals rapidly.

- In the subacute setting of ischemic stroke, there may not be a need to rapidly bring BP down to the targeted secondary stroke prevention range. lowering SBP to $140 \mathrm{~mm} \mathrm{Hg}$ in patients with $\mathrm{ICH}$ presenting with a SBP of $150-220 \mathrm{~mm} \mathrm{Hg}{ }^{\text {e11 }}$

\section{Antihypertensive agents in stroke}

As discussed, the need for rapid BP control in both AIS and ICH often requires IV agents. Such agents should be rapidly acting, be easy to titrate, and have few side effects and short half-lives. ${ }^{\text {e12 }}$ Some of the commonly used IV medications are nicardipine, labetalol, sodium nitroprusside, nitroglycerine, enalaprilat, and hydralazine. Sodium nitroprusside is not an ideal agent for acute reduction of BP due to its unpredictable dose-response relationship, risk of rebound hypertension, possibility of cyanide toxicity during prolonged use, and potential to cause raised intracranial pressure. ${ }^{\mathrm{el} 3}$ Although hydralazine is used frequently for acute reduction of BP, its use in routine clinical practice is limited due to its selective arteriolar vasodilator effect resulting in reflex tachycardia leading to myocardial injury. ${ }^{\mathrm{el1}}$

Recently, new data have been published investigating the use of different BP medications in the acute stroke setting. To compare the therapeutic response and tolerability of labetalol boluses vs IV nicardipine infusion, Liu-DeRyke et al. ${ }^{\text {el4 }}$ conducted a small pseudorandomized trial in the acute stroke setting $(\mathrm{n}=54$; 19 ischemic stroke, $29 \mathrm{ICH}, 6$ subarachnoid hemorrhage). Their findings demonstrated that a higher proportion of patients in the nicardipine group achieved goal BP within 60 minutes of treatment initiation $(100 \%$ vs $61 \%)$ and spent a greater amount of time in the goal BP range compared to the labetalol group. In addition, the number of dose adjustments required to reach goal $\mathrm{BP}$ was also lower (0 vs $2, p<0.001)$ in the nicardipine group, indicating a reliable dose response. While there are several limitations to this small, single-center trial, it is one of the first studies comparing 2 commonly used medications in the acute stroke setting.

Owing to its rapid onset of action, short half-life, and selective arterial vasodilator effect, clevidipine, a new calcium channel antagonist, is being increasingly studied in the critical care setting. ${ }^{\text {e15 }}$ The Evaluation of Patients with Acute Hypertension and Intracerebral Hemorrhage with Intravenous Clevidipine Treatment study tested the efficacy and safety of clevidipine in a multicenter, single-arm, open-label design that included spontaneous ICH patients presenting to the emergency department within 6 or 12 hours of symptom onset and with SBP $>160 \mathrm{~mm}$ Hg. ${ }^{\text {e16 }}$ Target BP was achieved in $96.9 \%$ of patients in a median time of 5.5 minutes. While the results of this study do not warrant a change in current clinical practice given that this was a pilot study involving a small cohort of patients, the ability to rapidly control high BP is notable and merits further study to determine whether this type of medication should be incorporated into the routine management of hypertension in the acute setting of stroke.

While IV agents are the mainstay of proficient BP management in the hyperacute stroke setting, oral agents are the cornerstone of $\mathrm{BP}$ control in the outpatient setting. Recent guidelines from the 8th Joint National Committee ${ }^{\mathrm{e} 77}$ (table) can serve as a foundation for clinicians to supplement their clinical expertise in achieving successful long-term outcomes for both primary and secondary stroke prevention goals.

\section{REFERENCES}

1. Gaciong Z, Siński M, Lewandowski J. Blood pressure control and primary prevention of stroke: summary of the recent clinical trial data and meta-analyses. Curr Hypertens Rep 2013;15:559-574. 
2. Lawes CM, Bennett DA, Feigin VL, Rodgers A. Blood pressure and stroke: an overview of published reviews. Stroke 2004;35:776-785.

3. Qureshi AI, Ezzeddine MA, Nasar A, et al. Prevalence of elevated blood pressure in 563,704 adult patients with stroke presenting to the ED in the United States. Am J Emerg Med 2007;25:32-38.

4. Kleindorfer D, Kissela B, Schneider A, et al. Eligibility for recombinant tissue plasminogen activator in acute ischemic stroke: a population-based study. Stroke 2004;35:e27-e29.

5. Fonarow GC, Smith EE, Saver JL, et al. Timeliness of tissue-type plasminogen activator therapy in acute ischemic stroke: patient characteristics, hospital factors, and outcomes associated with door-toneedle times within 60 minutes. Circulation 2011;123:750-758.

6. Saver JL, Fonarow GC, Smith EE, et al. Time to treatment with intravenous tissue plasminogen activator and outcome from acute ischemic stroke. JAMA 2013;309:2480-2488.

7. Skolarus LE, Scott PA, Burke JF, et al. Antihypertensive treatment prolongs tissue plasminogen activator door-to-treatment time: secondary analysis of the INSTINCT trial. Stroke 2012;43:33923394.

8. Jauch EC, Saver JL, Adams HP, et al. Guidelines for the early management of patients with acute ischemic stroke: a guideline for healthcare professionals from the American Heart Association/American Stroke Association. Stroke 2013;44:870-947.

9. An international randomized trial comparing four thrombolytic strategies for acute myocardial infarction: the GUSTO investigators. N Engl J Med 1993;329:673-682.

10. Comparison of invasive and conservative strategies after treatment with intravenous tissue plasminogen activator in acute myocardial infarction: results of the thrombolysis in myocardial infarction (TIMI) phase II trial: the TIMI Study Group. N Engl J Med 1989;320:618-627.

11. Ahmed N, Wahlgren N, Brainin M, et al. Relationship of blood pressure, antihypertensive therapy, and outcome in ischemic stroke treated with intravenous thrombolysis: retrospective analysis from Safe Implementation of Thrombolysis in Stroke-International Stroke Thrombolysis Register (SITS-ISTR). Stroke 2009;40:2442-2449.

12. de Los Ríos la Rosa F, Khoury J, Kissela BM, et al. Eligibility for intravenous recombinant tissue-type plasminogen activator within a population: the effect of the European Cooperative Acute Stroke Study (ECASS) III trial. Stroke 2012;43:1591-1595.

13. Sheth KN, Sims JR. Neurocritical care and periprocedural blood pressure management in acute stroke. Neurology 2012;79(suppl 1):S199-S204.

14. Martin-Schild S, Hallevi H, Albright KC, et al. Aggressive blood pressure-lowering treatment before intravenous tissue plasminogen activator therapy in acute ischemic stroke. Arch Neurol 2008;65: $1174-1178$.

15. Tarlov N, Nien YL, Zaidat OO, Nguyen TN. Periprocedural management of acute ischemic stroke intervention. Neurology 2012;79(suppl 1):S182-S191.

16. Patel VN, Gupta R, Horn CM, Thomas TT, Nogueira RG. The neuro-critical care management of the endovascular stroke patient. Curr Treat Options Neurol 2013;15:113-124.

17. Davis MJ, Menon BK, Baghirzada LB, et al. Anesthetic management and outcome in patients during endovascular therapy for acute stroke. Anesthesiology 2012;116:396-405.

18. Leslie-Mazwi TM, Sims JR, Hirsch JA, Nogueira RG. Periprocedural blood pressure management in neurointerventional surgery. J Neurointerv Surg 2011;3:66-73.

19. Saver JL. Time is brain: quantified. Stroke 2006;37:263-266.

20. Hadjiev DI, Mineva PP. Elevated blood pressure management in acute ischemic stroke remains controversial: could this issue be resolved? Med Hypotheses 2013;80:50-52.

21. Sandset EC, Bath PM, Boysen G, et al. The angiotensin-receptor blocker candesartan for treatment of acute stroke (SCAST): a randomised, placebo-controlled, double-blind trial. Lancet 2011;377:741-750.

22. He J, Zhang $\mathrm{Y}, \mathrm{Xu} \mathrm{T}$, et al. Effects of immediate blood pressure reduction on death and major disability in patients with acute ischemic stroke: the CATIS randomized clinical trial. JAMA 2014; 311:479-489.

23. Alqadri SL, Sreenivasan V, Qureshi AI. Acute hypertensive response management in patients with acute stroke. Curr Cardiol Rep 2013;15:426.

24. Delgado-Mederos R, Ribo M, Rovira A, et al. Prognostic significance of blood pressure variability after thrombolysis in acute stroke. Neurology 2008;71:552-558.

25. Aslanyan S, Fazekas F, Weir CJ, Horner S, Lees KR; Investigators GISCa. Effect of blood pressure during the acute period of ischemic stroke on stroke outcome: a tertiary analysis of the GAIN International Trial. Stroke 2003;34:2420-2425.

26. Benavente OR, Coffey CS, Conwit R, et al. Blood-pressure targets in patients with recent lacunar stroke: the SPS3 randomised trial. Lancet 2013;382:507-515.

27. Chimowitz MI, Lynn MJ, Howlett-Smith H, et al. Comparison of warfarin and aspirin for symptomatic intracranial arterial stenosis. N Engl J Med 2005;352:1305-1316. 
28. Turan TN, Cotsonis G, Lynn MJ, Chaturvedi S, Chimowitz M; Investigators W-ASIDWT. Relationship between blood pressure and stroke recurrence in patients with intracranial arterial stenosis. Circulation 2007;115:2969-2975.

29. Derdeyn CP, Chimowitz MI, Lynn MJ, et al. Aggressive medical treatment with or without stenting in high-risk patients with intracranial artery stenosis (SAMMPRIS): the final results of a randomised trial. Lancet 2014;383:333-341.

30. Powers WJ, Zazulia AR, Videen TO, et al. Autoregulation of cerebral blood flow surrounding acute (6 to 22 hours) intracerebral hemorrhage. Neurology 2001;57:18-24.

\section{ACKNOWLEDGMENT}

The authors thank Dr. Karen Albright and Dr. P. Roc Chen for editorial support.

\section{STUDY FUNDING}

No targeted funding reported.

\section{DISCLOSURES}

R. Bowry and D.D. Navalkele report no disclosures. N.R. Gonzales serves as an editorial board member for Stroke and receives research support from the NIH/National Institute of Neurological Disorders and Stroke. Full disclosure form information provided by the authors is available with the full text of this article at Neurology.org/cp.

\section{Related articles from other AAN physician and patient resources}

\section{Neurology ${ }^{\circledR}$ - Neurology.org}

Lower stroke risk with lower blood pressure in hemodynamic cerebral ischemia March 25, 2014;82:1027-1032.

Blood pressure and cerebral ischemia: A continuing dilemma March 25, 2014;82:1018-1019.

\section{Continuum ${ }^{\circledR}$ - ContinuumJournal.com}

Blood Pressure Reduction in Secondary Stroke Prevention December 2011;1233-1241.

Risk Factor Management for Stroke Prevention April 2014;296-308.

\section{Neurology Now ${ }^{\circledR} \quad$ Neurologynow.com}

Simple Stroke Prevention Tips: Seven proven strategies to slash your risk of brain attack August/September 2014;10:43-45.

Control Your Blood Pressure, Save Your Brain

June/July 2013;9:38-42. 